\title{
Increased osteopontin expression in endometrial carcinoma is associated with better survival outcome
}

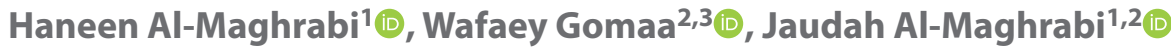 \\ ${ }^{1}$ Department of Pathology, King Faisal Specialist Hospital and Research centre, Jeddah, Saudi Arabia \\ ${ }^{2}$ Department of Pathology, Faculty of Medicine, King Abdulaziz University, Jeddah, Saudi Arabia \\ ${ }^{3}$ Department of Pathology, Faculty of Medicine, Minia University, Al-Minia, Egypt
}

\begin{abstract}
Objectives: Osteopontin (OPN) is a key extracellular matrix protein that is involved in cancer progression. The aim of the current study is to investigate the relation of OPN immunostaining in endometrial carcinoma with clinicopathological parameters.

Material and methods: Archival 71 endometrial carcinomas and 30 non-neoplastic endometrial tissues were obtained from the Department of Pathology at King Abdulaziz University Jeddah, Saudi Arabia. Tissue microarrays were constructed. Tissue sections were stained using anti-human OPN monoclonal antibody. Immunostaining results were recorded and analysed.

Results: In non-neoplastic endometrial tissues, high (increased) OPN immunostaining was observed in $100 \%$. In endometrial carcinoma, high (increased) OPN immunostaining was seen in $64.8 \%$ of cases. High (increased) OPN immunostaining was more frequent in non-neoplastic tissues than in endometrial carcinoma $(p<0.001)$. OPN immunostaining showed no association with histological type, FIGO tumour grade, tumour size, myometrial invasion, lymphovascular invasion, surgical resection margin or lymph node metastasis. On the other hand, high (increased) OPN immunostaining was associated with better overall survival [Log Rank (Mantel-Cox) $=4.385, p=0.003$ ].

Conclusions: In endometrial carcinoma, immunohistochemical staining of OPN could be a helpful tool in the prediction survival pattern. OPN immunostaining showed no association with most clinicopathological features. Further investigations both clinical and molecular are needed to explore the downstream of OPN in endometrial carcinoma.
\end{abstract}

Key words: endometrial carcinoma; tissue microarray; immunohistochemistry; osteopontin

Ginekologia Polska 2020; 91, 2: 73-78

\section{INTRODUCTION}

Endometrial carcinoma (EC) is one of the most common malignant tumours of female genital system. While early diagnosed stages of EC can be cured by surgical resection only, advanced cases of EC can be complicated by distant organ metastasis and peritoneal involvement associated with poor patient free survival rate. Thus, understating the genetic pathophysiology pathway of EC play a crucial role in therapeutic management protocol of the disease [1]. Angiogenesis of a tumour is associated with genes expression in human endometrial endothelial cells (HEECs) which can enhance the process of angiogenesis, increase tumour permeability, rapid cell proliferation and increase metastatic rate.

Osteopontin (OPN) was first discovered as one of CD44 ligands [2]. It is a 70-KDa particle of phosphorylated N-linked glycoprotein, which was primarily discovered in bone matrix. OPN can be found in the epithelial linings of salivary glands, sweat ducts, breast, bronchi, pancreas, gall bladder, urinary bladder, and reproductive systems [3]. OPN is a glycosylated phosphoprotein of the extracellular matrix particles, which contains nine consecutive aspartic acid residues, considered a highly acidic region named $\mathrm{N}$-terminal signal [4]. It can be also detected in activated defence cells like macrophages, lymphocytes and leukocytes [5]. OPN has the capacity to bind with osteocalcin, type I collagen, and fibronectin [6]. It is also involved in many precise cellular processes like cellular adhesions, tumourigenesis, angiogenesis, and tumours distant metastasis. Moreover, its overexpression can affect transduction of PI3 Kinase/AKT activation signalling pathway in various tumours which affect the cellular apoptosis [7]. 
OPN overexpression is one of the phospho-glycoprotein (P-gp) with oncogenic potential in various body tumours $[8,9]$. Many studies discussed the role of OPN in various epithelial malignancies such as breast, colon cancer, thyroid and melanoma [10-17].

However, more studies are needed to enlighten the role of OPN expression in EC. The aim of this study is to find out the relation OPN immunostaining with clinical and pathological features of EC based tissue microarray.

\section{MATERIAL AND METHODS}

\section{Patients}

The study utilised paraffin wax tumour blocks from 71 patients diagnosed with endometrial carcinoma in the period from 2003-2012. Also paraffin blocks from 30 non-neoplastic endometrial tissues in the period from 1995-1998 (20 proliferative endometrium and 10 secretory endometrium). All blocks were used from the archives of the Department of Pathology at King Abdulaziz University, Jeddah, Saudi Arabia. Some clinicopathological characteristics of patients are listed in Table 1. For statistical purpose, FIGO stages were classified into limited to uterine corpus (FIGO Stage I and II) and beyond the uterine corpus (FIGO III and IV). Also grades were reclassified as low grade (grade I) and (grades II and III). Data is shown in Table 1. The study was done following rules of the ethics committee of Faculty of Medicine, King Abdulaziz University, Saudi Arabia, and declaration of Helsinki.

\section{Tissue Microarray}

Archival paraffin-embedded endometrial carcinoma samples and neoplastic tissues were selected and the desired areas were marked on haematoxylin and eosin (H\&E)-stained slides. Arrays were produced by retrieving cores $(1.5 \mathrm{~mm}$ in diameter each) from marked areas of each 'donor' tissue block and brought into new recipient paraffin blocks. Tissue microarrays were constructed in an automated tissue microarrayer [TMA Master 1.14 SP3 (3D Histech Ltd. Budapest, Hungary)]. Placenta tissue was used for orientation [17, 18].

\section{Immunohistochemistry}

The constructed tissue microarray paraffin blocks were sliced at $4 \mu \mathrm{m}$. Tissues were mounted on positive-charged slides (Leica Microsystems Plus Slides). In an automated immunostained BenchMark XT, Ventana ${ }^{\circledR}$ Medical systems Inc., Tucson, AZ, USA) immunohistochemistry was performed. Slides were deparaffinised in xylene and rehydrated. Slides were incubated with pre-diluted CC1 (cell conditioning solution) for 60 minutes to perform pre-treatment. Polyclonal anti-human rabbit anti-osteopontin antibody (Spring ${ }^{\mathrm{TM}}$ Bioscience; Cat \# E3284) was incubated at $37^{\circ} \mathrm{C}$ for 20 minutes. The detection kit was used from Ventana I-view DAB. The slides were washed and Mayer's haematoxylin was used as a counter stain. The appropriate negative and positive control slides were used.

\section{Interpretation of OPN Immunostaining}

The immunostained slides were examined by two pathologists (HM, WG) and a semiquantitative approach was used including the percentage of OPN positive cells. The percentage of OPN positivity was scaled as follows; (1) $0-25 \%$,

\begin{tabular}{|c|c|c|}
\hline \multicolumn{2}{|l|}{ Parameter } & Number (\%) \\
\hline \multirow{2}{*}{ Age } & $<60$ years & $49(69 \%)$ \\
\hline & $>60$ years & $22(31 \%)$ \\
\hline \multirow{2}{*}{ Histological type } & Endometrioid & $66(93 \%)$ \\
\hline & Serous & $5(7 \%)$ \\
\hline \multirow{3}{*}{ FIGO tumour grade } & Grade 1 & $44(62 \%)$ \\
\hline & Grade 2 & $16(22.5 \%)$ \\
\hline & Grade 3 & $11(15.5 \%)$ \\
\hline \multirow{2}{*}{ Tumour size } & $\leq 2 \mathrm{~cm}$ & $35(49.3 \%)$ \\
\hline & $>2 \mathrm{~cm}$ & $36(50.7 \%)$ \\
\hline \multirow{2}{*}{ Myometrial invasion } & $<50 \%$ & $57(80.3 \%)$ \\
\hline & $\geq 50 \%$ & $14(19.7 \%)$ \\
\hline \multirow{2}{*}{ Lymphovascular } & Absent & $68(95.8 \%)$ \\
\hline & Present & $3(4.2 \%)$ \\
\hline \multirow{2}{*}{ Surgical resection margin } & Free & $67(94.4 \%)$ \\
\hline & Involved & $4(5.6 \%)$ \\
\hline \multirow{3}{*}{ Lymph node metastasis } & Absent & $33(46.5 \%)$ \\
\hline & Present & $4(5.6 \%)$ \\
\hline & Not sampled & $34(47.9 \%)$ \\
\hline \multirow{4}{*}{ FIGO Staging } & I & $51(71.8 \%)$ \\
\hline & II & $7(9.9 \%)$ \\
\hline & III & $7(9.9 \%)$ \\
\hline & IV & $6(8.5 \%)$ \\
\hline \multirow{2}{*}{ Local Recurrence } & Absent & $60(84.5 \%)$ \\
\hline & Present & $11(15.5 \%)$ \\
\hline
\end{tabular}

FIGO (International Federation of Gynaecology and Obstetrics) Stage I - tumour confined to corpus uteri

IA - tumour limited to endometrium or invades less than one-half of the myometrium

IB - tumour invades one-half or more of the myometrium Stage II - tumour invades stromal connective tissue of the cervix but does not extend beyond uterus

Stage III - there is regional tumour spread.

IIIA - tumour involves serosa and/or adnexa (direct extension or metastasis)

IIIB - vaginal involvement (direct extension or metastasis) or parametrial involvement

IIIC - the tumour involves regional lymph nodes

IIIC1 - regional lymph node metastasis to pelvic lymph nodes

IIIC2 - Regional lymph node metastasis to para-aortic lymph nodes, with or without positive pelvic lymph nodes

Stage IV - the tumour invades contiguous organs or has metastasized to remote organ sites

IVA - tumour invades bladder mucosa and/or bowel mucosa (bullous oedema is not sufficient to classify a tumour as T4)

$\mathrm{VB}$ - distant metastasis 


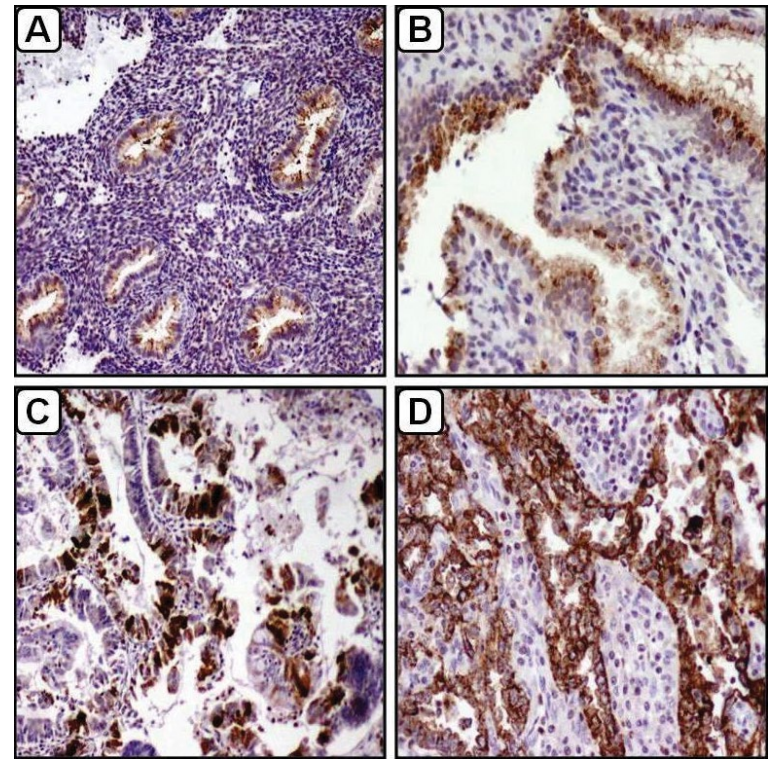

Figure 1. Immunostaining of OPN in non-neoplastic endometrium and endometrial carcinoma

OPN is shown in the cytoplasm of proliferative endometrium

(A-100X), secretory endometrium (B-200X), well-differentiated endometrial carcinoma (C-200X), and in moderately differentiated endometrial carcinoma (D-200X). Immunohistochemical labelling was done using the anti-OPN antibody and diaminobenzidine used as the chromogen and haematoxylin as counterstain.

\begin{tabular}{|c|c|c|}
\hline & $\begin{array}{l}\text { Endometrial } \\
\text { carcinoma } \\
(\mathbf{n}=\mathbf{7 1})\end{array}$ & $\begin{array}{l}\text { Non-neoplastic } \\
\text { endometrium } \\
(n=30)\end{array}$ \\
\hline Low (decreased) immunostaining & $25(35.2 \%)$ & $0(0 \%)$ \\
\hline High (increased) immunostaining & $46(64.8 \%)$ & $30(100 \%)$ \\
\hline$p$ value & $<0.001^{*}$ & $<0.001^{*}$ \\
\hline
\end{tabular}

*One sample non-parametric chi-square test

(2) $26-50 \%$, (3) $50-100 \%$. For cytoplasmic immunostaining; 3 (heavy and intense brown immunostaining), 2 (brown immunostaining lighter than 3), 1 (brown immunostaining is weak), and 0 (no brown immunostaining). The result of summing percentage and intensity provided scores from 1-6. For the statistical analysis, an OPN immunostaining score of 1-3 was considered as low (decreased) immunostaining, and an OPN immunostaining score of 4-6 was considered as high (increased) immunostaining [19].

\section{Statistical analysis}

To study the variation between two groups of patients on one variable, the Mann Whitney test was used. Non-parametric chi-square was used to test variance along one variable. The survival differences and probabilities and the Log Rank test was tested by using the univariate Kaplan-Meier procedure was used. The end-point for patients was last seen or death. The model was controlled for confounding variables. Statistical procedures were performed using SPSS ${ }^{\circ}$ Release 16.0. Statistical significance was determined at $p$ value of $\leq 0.05$ and was 2 -sided.

\section{RESULTS \\ Pattern of OPN immunostaining}

Immunostaining of OPN was indicated by cytoplasmic brown colour in non-neoplastic and neoplastic endometrial (Fig. 1). In non-neoplastic endometrial tissues, high (increased) immunostaining was observed in all specimens (100\%) while in EC was $98.5 \%$. The incidence of increased OPN (high immunostaining) immunostaining was more frequent in non-neoplastic tissues (100\%) than in endometrial carcinoma (64.9\%) ( $p<0.001)$. In endometrial carcinoma, high (increased) OPN immunostaining was seen in $71.8 \%$. The occurrence of high (increased) OPN immunostaining was statistically more than low (decreased) immunostaining ( $p \leq 0.001$ ). Data is shown in Table 2.

\section{Correlation of OPN immunostaining with clinicopathological features of EC}

The present study revealed that there was no correlation between OPN immunostaining with most clinicopathological features (data is represented in Table 3).

\section{Correlation of OPN immunostaining with survival outcomes}

On the other hand, high (increased) OPN immunostaining in endometrial carcinoma was associated with better survival outcomes for overall survival (log-rank $=4.385$, $p=0.003$ ) (Fig. 2).

\section{DISCUSSION}

EC affects approximately 2-3\% women worldwide [7]. The process of tumorigenesis in EC must be studied and understood in order to establish proper tumour management under convenient designed therapeutic standards. Previous reports stated the possible role of OPN in tumour progression and metastasis [20,21].

Previously, reported that OPN is produced in higher levels in endometrial tumour cells than normal non metastatic cells. OPN particles can negatively affect the consecutive pathophysiology process of cellular adhesion, cellular migration, and invasion by av $\beta 3$ receptors [7, 22]. Also, OPN can activate ERK $1 / 2$ and PI3K/ AKT signalling pathway leading to promotion in cellular migration, proliferation, and invasion due to increased MMP-2 expression [7]. Indeed, OPN can intercede in cell adhesion, and tumour colonies formation. The role of OPN is important to be studied as a prognostic marker in EC that might contribute to future 
Table 3. Correlation between Clinicopathological features and OPN Immunostaining in tumours $(n=71)$

\section{Parameter}

Age

Histological type

FIGO tumour grade

Tumour size

Myometrial invasion

Lymphovascular

Surgical resection margin

Lymph node metastasis

FIGO Staging

Local Recurrence

*Mann Whitney test

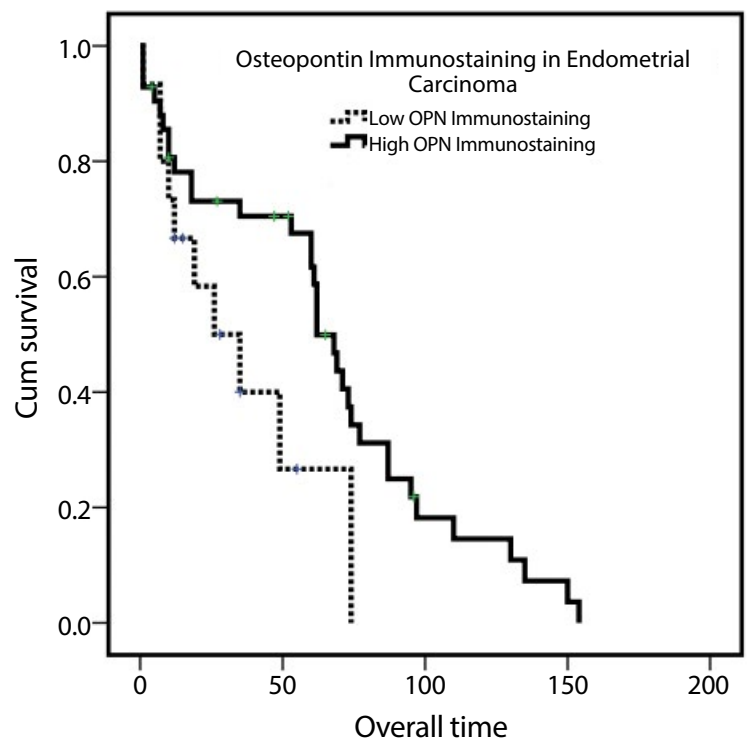

Figure 2. Overall survival curve (Kaplan Meier) according to OPN immunostaining

High (increased) OPN immunostaining is associated with better overall survival [Log Rank (Mantel-Cox) $=4.385, p=0.003$ ]

therapeutic management of the disease. Only a few papers in the literature correlate OPN expression and its clinico- pathological significant with EC. Some results were inconclusive due to limited sample size.

In the present study, the immunostaining of OPN in normal endometrial tissue and in EC was carried on tissue microarray format to find out its correlation with clinicopathological features and possible clinical implications on the disease. In non-neoplastic endometrial tissues, high (increased) OPN immunostaining was observed in all specimens (100\%). The immunolocalisation of OPN in normal endometrial tissue was reported before as strong cytoplasmic staining in both proliferative and secretory phases. This finding was attributed to the role of OPN in normal cell regulation of menstrual cycle process and maternal-foetal interface reaction during foetal placental implantation [22-25]. We found that high (increased) OPN immunostaining was found in 46 EC (64.8\%), while low (decreased) OPN was reported in 25 cases (35.2\%). On the other hand, these studies reported that OPN is decreased in malignant tissue, but still detected in about half of tumours [7, 23, 26-28].

In the current study, we could not establish any statistically significant association between OPN immunostaining and the clinicopathological of EC. One of the few studies of OPN in EC reported similar findings [28]. However, in our study increased OPN immunostaining was reported in 
31 of low grade EC (43.6\%) while in 15 high grade EC (21.1\%) $(\mathrm{p}=0.184)$. A previous report could not establish any relation between OPN and EC grade [28]. In a previous study, OPN immunostaining was shown to be increased with a higher tumour grade [23]. On the contrary, it was reported that high levels of OPN was associated with more differentiated tumours suggesting a protective role of OPN in EC [26].

In the present study, EC that invade less than $50 \%$ of myometrial thickness were positive in $80.3 \%$, compared to a lower percentage $(19.7 \%)$ in tumours invadeing more than $50 \%$ of myometrial thickness $(p=0.168)$. Although statistically not significant, however there is a trend that increased OPN immunostaining may be associated with less invading tumours. A study reported that high OPN is associated with lower stages [23]. In previous reports, silencing of OPN mRNA led to reduced invasion of EC cell lines in vitro and reduction of tumour size [1, 8, 29]. The statistical results from our study are not supporting this finding as there was no relation between OPN immunostaining and tumour stage or tumour size. In the current study, increased OPN immunostaining is significantly associated with higher overall survival probabilities. This result is in accordance with a previous report [26].

All together, our findings may support the trend that increased OPN immunostaining in EC may have good prognostic outcomes. However, the conflicting results with the few reports may be due to different sample size, technical issues, and/or different cut-off point in immunohistochemistry interpretation. Therefore, for future consideration to stratify EC using OPN as prognostic molecular marker and therapeutic target, it has to be studied in the context of its multiple functions and different isoforms. Our study has a limitation of a relatively small number of patients and, therefore, should be expanded to confirm these findings.

\section{CONCLUSIONS}

Our results showed that increased OPN immunostaining staining is considered a good predictor factor for survival outcome in EC and may have a role of future therapeutic management. The role of OPN as a molecular marker in EC is still unclear and needs to be established as it may be a possible target for future therapeutic applications in EC.

\section{REFERENCES}

1. Du Xl, Jiang T, Sheng Xg, et al. Inhibition of osteopontin suppresses in vitro and in vivo angiogenesis in endometrial cancer. Gynecol Oncol. 2009; 115(3): 371-376, doi: 10.1016/j.ygyno.2009.08.029, indexed in Pubmed: 19783287.

2. Smyth NA, Murawski CD, Adams SB, et al. International Consensus Group on Cartilage Repair of the Ankle. Osteochondral Allograft: Proceedings of the International Consensus Meeting on Cartilage Repair of the Ankle. Foot Ankle Int. 2018; 39(1_suppl): 35S-40S, doi: 10.1177/1071100718781097, indexed in Pubmed: 30215308.

3. Brown LF, Berse B, Van de Water $L$, et al. Expression and distribution of osteopontin in human tissues: widespread association with lu- minal epithelial surfaces. Mol Biol Cell. 1992; 3(10): 1169-1180, doi: 10.1091/mbc.3.10.1169, indexed in Pubmed: 1421573.

4. Ritter NM, Farach-Carson MC, Butler WT. Evidence for the formation of a complex between osteopontin and osteocalcin. J Bone Miner Res. 1992; 7(8): 877-885, doi: 10.1002/jbmr.5650070804, indexed in Pubmed: 1442202.

5. Rodrigues LR, Teixeira JA, Schmitt FL, et al. The role of osteopontin in tumor progression and metastasis in breast cancer. Cancer Epidemiol Biomarkers Prev. 2007; 16(6): 1087-1097, doi: 10.1158/1055-9965.EPI06-1008, indexed in Pubmed: 17548669.

6. Philip S, Bulbule A, Kundu GC. Osteopontin stimulates tumor growth and activation of promatrix metalloproteinase-2 through nuclear factor-kappa B-mediated induction of membrane type 1 matrix metalloproteinase in murine melanoma cells. J Biol Chem. 2001; 276(48): 44926-44935, doi: 10.1074/jbc.M103334200, indexed in Pubmed: 11564733.

7. Li Y, Xie Y, Cui D, et al. Osteopontin Promotes Invasion, Migration and Epithelial-Mesenchymal Transition of Human Endometrial Carcinoma Cell HEC-1A Through AKT and ERK1/2 Signaling. Cell Physiol Biochem. 2015; 37(4): 1503-1512, doi: 10.1159/000438518, indexed in Pubmed: 26509489.

8. Hahne JC, Meyer SR, Kranke P, et al. Studies on the role of osteopontin-1 in endometrial cancer cell lines. Strahlenther Onkol. 2013; 189(12): 10401048, doi: 10.1007/s00066-013-0434-y, indexed in Pubmed: 24126938.

9. Senger $D$, Wirth $D$, Hynes R. Transformed mammalian cells secrete specific proteins and phosphoproteins. Cell. 1979; 16(4): 885-893, doi: 10.1016/0092-8674(79)90103-x.

10. Assidi M, Gomaa W, Jafri M, et al. Prognostic value of Osteopontin (SPP1) in colorectal carcinoma requires a personalized molecular approach. Tumour Biol. 2019; 41(9): 1010428319863627, doi: 10.1177/1010428319863627, indexed in Pubmed: 31500540.

11. Furger KA, Menon RK, Tuck AB, et al. The functional and clinical roles of osteopontin in cancer and metastasis. Curr Mol Med. 2001; 1(5): 621-632, doi: 10.2174/1566524013363339, indexed in Pubmed: 11899236.

12. Ue T, Yokozaki H, Kitadai $Y$, et al. Co-expression of osteopontin and CD44v9 in gastric cancer. Int J Cancer. 1998; 79(2): 127-132, doi: 10.1002/ (sici) 1097-0215(19980417)79:2<127::aid-ijc5>3.0.co;2-v, indexed in Pubmed: 9583725.

13. Chambers AF, Wilson SM, Kerkvliet N, et al. Osteopontin expression in lung cancer. Lung Cancer. 1996; 15(3): 311-323, doi: 10.1016/01695002(95)00595-1, indexed in Pubmed: 8959677.

14. Tuck $A B, O^{\prime}$ Malley FP, Singhal $H$, et al. Osteopontin expression in a group of lymph node negative breast cancer patients. Int J Cancer. 1998; 79(5): 502-508, doi: 10.1002/(sici) 1097-0215(19981023)79:5<502::a id-ijc10>3.0.co;2-3, indexed in Pubmed: 9761120.

15. Rittling SR, Chambers AF. Role of osteopontin in tumour progression. $\mathrm{Br}$ J Cancer. 2004; 90(10): 1877-1881, doi: 10.1038/sj.bjc.6601839, indexed in Pubmed: 15138464

16. Gomaa W, Al-Ahwal M, Hamour O, et al. Osteopontin cytoplasmic immunoexpression is a predictor of poor disease-free survival in thyroid cancer. Journal of Microscopy and Ultrastructure. 2013; 1(1): 8, doi: 10.1016/j.jmau.2013.07.001.

17. Al-Maghrabi J, Emam E, Gomaa W, et al. c-MET immunostaining in colorectal carcinoma is associated with local disease recurrence. BMC Cancer. 2015; 15: 676, doi: 10.1186/s12885-015-1662-6, indexed in Pubmed: 26459369.

18. Gomaa W, Ke Y, Fujii H, et al. Tissue microarray of head and neck squamous carcinoma: validation of the methodology for the study of cutaneous fatty acid-binding protein, vascular endothelial growth factor, involucrin and Ki-67. Virchows Arch. 2005; 447(4): 701-709, doi: 10.1007/s00428-005-0002-7, indexed in Pubmed: 16012850.

19. Gomaa W, Al-Ahwal M, Hamour O, et al. Osteopontin cytoplasmic immunoexpression is a predictor of poor disease-free survival in thyroid cancer. Journal of Microscopy and Ultrastructure. 2013; 1(1): 8, doi: 10.1016/j.jmau.2013.07.001.

20. Wei R, Wong JP, Kwok HF. Osteopontin -- a promising biomarker for cancer therapy. J Cancer. 2017; 8(12): 2173-2183, doi: 10.7150/jca.20480, indexed in Pubmed: 28819419.

21. Castello LM, Raineri D, Salmi L, et al. Osteopontin at the Crossroads of Inflammation and Tumor Progression. Mediators Inflamm. 2017; 2017: 4049098, doi: 10.1155/2017/4049098, indexed in Pubmed: 28769537.

22. von Wolff M, Strowitzki T, Becker V, et al. Endometrial osteopontin, a ligand of beta3-integrin, is maximally expressed around the time of the "implantation window". Fertil Steril. 2001; 76(4): 775-781, doi: 10.1016/s0015-0282(01)02015-5, indexed in Pubmed: 11591413. 
23. Briese J, Schulte HM, Bamberger CM, et al. Expression pattern of osteopontin in endometrial carcinoma: correlation with expression of the adhesion molecule CEACAM1. Int J Gynecol Pathol. 2006; 25(2): 161-169, doi: 10.1097/01.pgp.0000189243.49522.ae, indexed in Pubmed: 16633066.

24. Casals G, Ordi J, Creus M, et al. Osteopontin and alphavbeta3 integrin as markers of endometrial receptivity: the effect of different hormone therapies. Reprod Biomed Online. 2010; 21(3): 349-359, doi: 10.1016/j. rbmo.2010.04.012, indexed in Pubmed: 20638909.

25. Casals G, Ordi J, Creus M, et al. Osteopontin and alphavbeta3 integrin expression in the endometrium of infertile and fertile women. Reprod Biomed Online. 2008; 16(6): 808-816, doi: 10.1016/s1472-6483(10)601460 , indexed in Pubmed: 18549690.

26. Cho $\mathrm{H}$, Kang ES, Kim YT, et al. Diagnostic and prognostic impact of osteopontin expression in endometrial cancer. Cancer Invest. 2009;
27(3): 313-323, doi: 10.1080/07357900802375738, indexed in Pubmed: 19194826.

27. Lax SF. Molecular genetic pathways in various types of endometria carcinoma: from a phenotypical to a molecular-based classification. Virchows Arch. 2004; 444(3): 213-223, doi: 10.1007/s00428-003-0947-3, indexed in Pubmed: 14747944

28. Hashiguchi $Y$, Tsuda H, Bandera CA, et al. Comparison of osteopontin expression in endometrioid endometrial cancer and ovarian endometrioid cancer. Med Oncol. 2006; 23(2): 205-212, doi: 10.1385/MO:23:2:205, indexed in Pubmed: 16720920.

29. Ramachandran S, Kwon KY, Shin SJ, et al. Regulatory role of osteopontin in malignant transformation of endometrial cancer. Mol Biol Rep. 2013; 40(5): 3623-3629, doi: 10.1007/s11033-012-2436-8, indexed in Pubmed: 23269624. 